\title{
Al Dente - Führungsreihe in die Welt der Zähne
}

\author{
Hilke Steinecke \& Peter Schubert
}

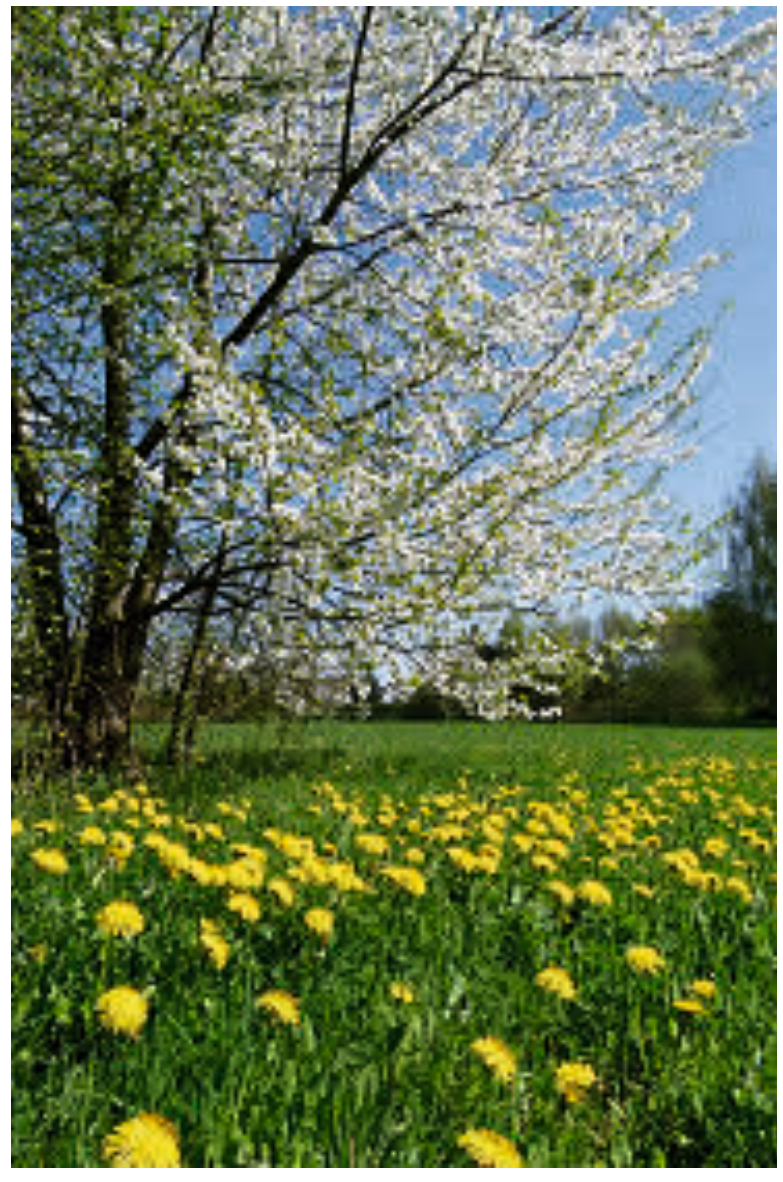

\section{Zähne in Pflanzennamen}

Eine neue abendliche Führungsreihe im Palmengarten befasste sich mit Zähnen und ihrer Beziehung zur Pflanzenwelt. Auf einem Rundgang durch das Freiland sowie durch die Gewächshäuser des Tropicariums wurde etlichen Pflanzen „auf den Zahn gefühlt“. Fachliche Informationen wurden dabei mit „bissigen Literaturzitaten“ oder „zahnigen Rezepten“ kombiniert. Es wurden zunächst einige Gewächse vorgestellt, die Zähne in ihrem Namen tragen, darunter der weit bekannte Löwenzahn (Taraxacum officinale) mit seinen typisch gezähnten Blättern. Nicht zu verwechseln ist diese Staude mit der ebenfalls als Löwenzahn oder aber auch Milchkraut bezeichneten Gattung Leontodon. Dem Bergwanderer begegnen diese gelb blühenden Korbblütler wie beispielsweise der Schweizer Löwenzahn ( $L$. helveticus) oft in großen Beständen in den Alpen auf Almwiesen und Matten. Auf die mit zwei Zähnen versehenen Früchte bezieht sich die Gattungsbezeichnung Zweizahn (Bidens). Zweizahn-Sorten sind beliebte Sommerblumen, $B$. pilosa oder B. tripartita beispielsweise kommen bei uns wild in Ufernähe vor. Der Pappus ist zu zwei steifen Zähnen reduziert. Mit ihrer Hilfe bleiben die Früchte im Fell von Tieren hängen und werden auf diese Art und Weise ausgebreitet. Die Blüten des Hohlzahns (Galeopsis) haben auf ihrer Unterlippe zwei hohle Ausstülpungen. Die an einen Hundezahn erinnernde Zwiebel führte zu dem $\mathrm{Na}$ men Hundszahnlilie (Erythronium dens-canis). Unter den Orchideen ist Odontoglossum als Zimmerpflanze beliebt. Die wörtliche Übersetzung des Gattungsnamens bedeutet Zahnzunge.

Gemäß der Signaturlehre wurde früher vielen Pflanzen, die an menschliche Organe oder andere Körperteile erinnern, eine entsprechende Heilwirkung zugeschrieben. Beim Zahntrost (Odontites rubra), einem Halbschmarotzer, ähnelt der Blütenstand mit seinen mehreren weißlichen, kleinen Blüten an eine Zahnreihe. Es ist es auch denkbar, dass die Blatt-Zähne mit den Zähnen des Menschen verglichen wurden. Extrakte aus dem Kraut wurden dementsprechend zur Linderung von Zahnschmerzen eingenommen. Zahnbohnen, die Samen von Pfingstrosen, sollten gegen Zahnschmerzen helfen. Zur Zahnpflege tragen Zahnstocher und Zahnbürste bei. Auch hierfür sind Kräuter gewachsen wie der lokal auch unter Zahnbürstenblume bekannte Schlangen-Knöterich (Polygonum bistorta) oder das Zahnstocherkraut (Ammi visnaga). Von letzterem wurden die Strahlen der doldigen Blütenstände tatsächlich als Zahnstocher verwendet.

Bei Zähnen und Pflanzen gibt es viele begriffliche Übereinstimmungen, wenn ihr Bau be- 
schrieben wird. So haben Zähne Wurzeln und Krone wie auch die Bäume. Pulpa bezeichnet in der Zahnkunde das Zahnmark, in der Botanik ein weiches Fruchtfleisch, wie es beispielsweise in der Kakaofrucht gut ausgeprägt ist.

\section{Vielfalt der Pflanzenzähne}

Die große Formenvielfalt der Zähne im Pflanzen- und Tierreich wurde an verschiedenen Stationen demonstriert. Gezähnt sind die Zungenblüten vieler Korbblütler oder die Ränder von Blättern. Die Art der Bezahnung ist oft ein wichtiges Bestimmungsmerkmal. Auf den ersten Blick sehen sich z. B. die Blätter von Hainbuche (Carpinus betulus) und Rotbuche (Fagus sylvatica) recht ähnlich, wobei die Rotbuche ganzrandige Blätter hat und der Rand von Hainbuchenblättern gezähnt ist. Zähne sind manchmal extrem scharf und wehrhaft, um Fressfeinde abzuwehren, wie die Blattstiele verschiedener Palmblätter (z. B. Hyphaene thebaica oder Livistona chinensis) beweisen. Auf den wehrhaften Blattstiel bezieht sich auch der Name Sägezahnpalme für Serenoa repens. Haken, Borsten oder Zähne an Früchten dienen meist dem Befestigen im Fell von Tieren, um die Samen möglichst effektiv ohne eigene Kraft auszubreiten.

\section{Zähne der Tiere}

Je nachdem, ob es sich um Pflanzen- oder Fleischfresser handelt, sind Tierzähne ganz unterschiedlich geformt, wie an verschiedenen Stellen der Führung erläutert wurde. Bei einigen Tieren dienen die Zähne nicht nur zum Kauen und Beißen, sondern auch zum Kämpfen, Imponieren oder Graben, wie Elefantenstoßzähne. Das Gürteltier ist mit 104 Zähnen Rekordhalter landlebender Säugetiere, im

Abb. 1 (Seite 138): Löwenzahn; der Name bezieht sich auf die gezähnten Blätter.

Abb. 2 (oben): Hohlzahn (Galeopsis segetum).

Abb. 3 (Mitte): Gezähnte Zungenblüten im Köpfchen der Wegwarte (Cichorium intybus).

Abb. 4 (unten): Zahnförmige Zwiebel der Hundszahnlilie (Erythronium dens-canis).
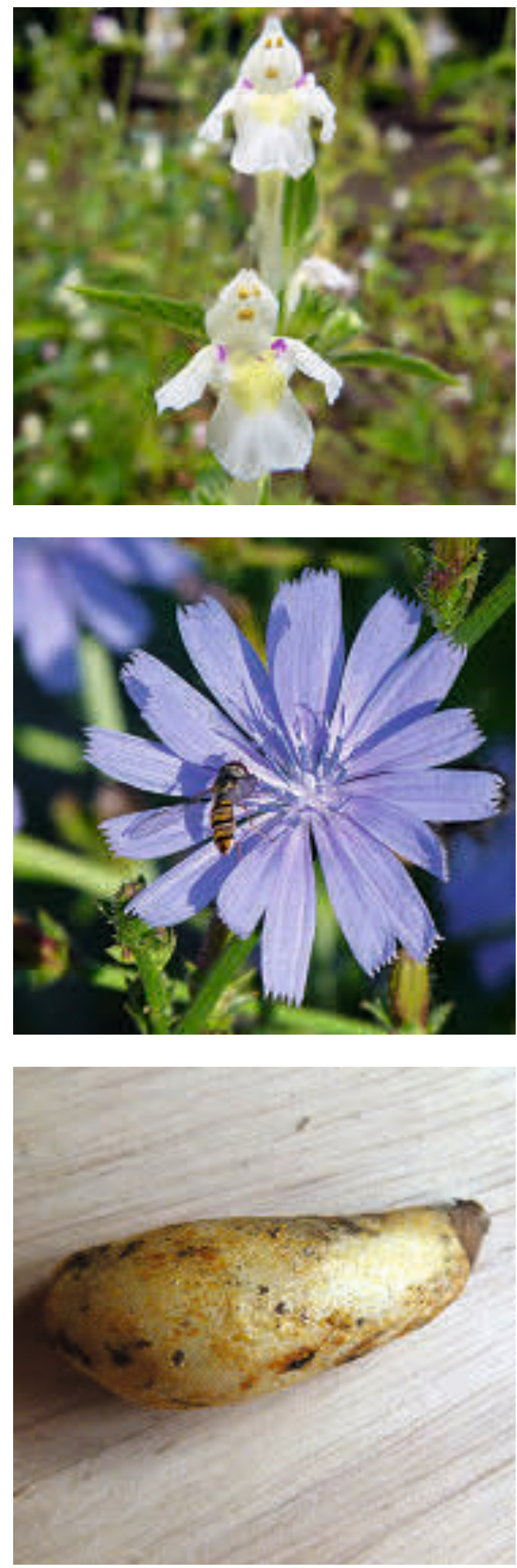


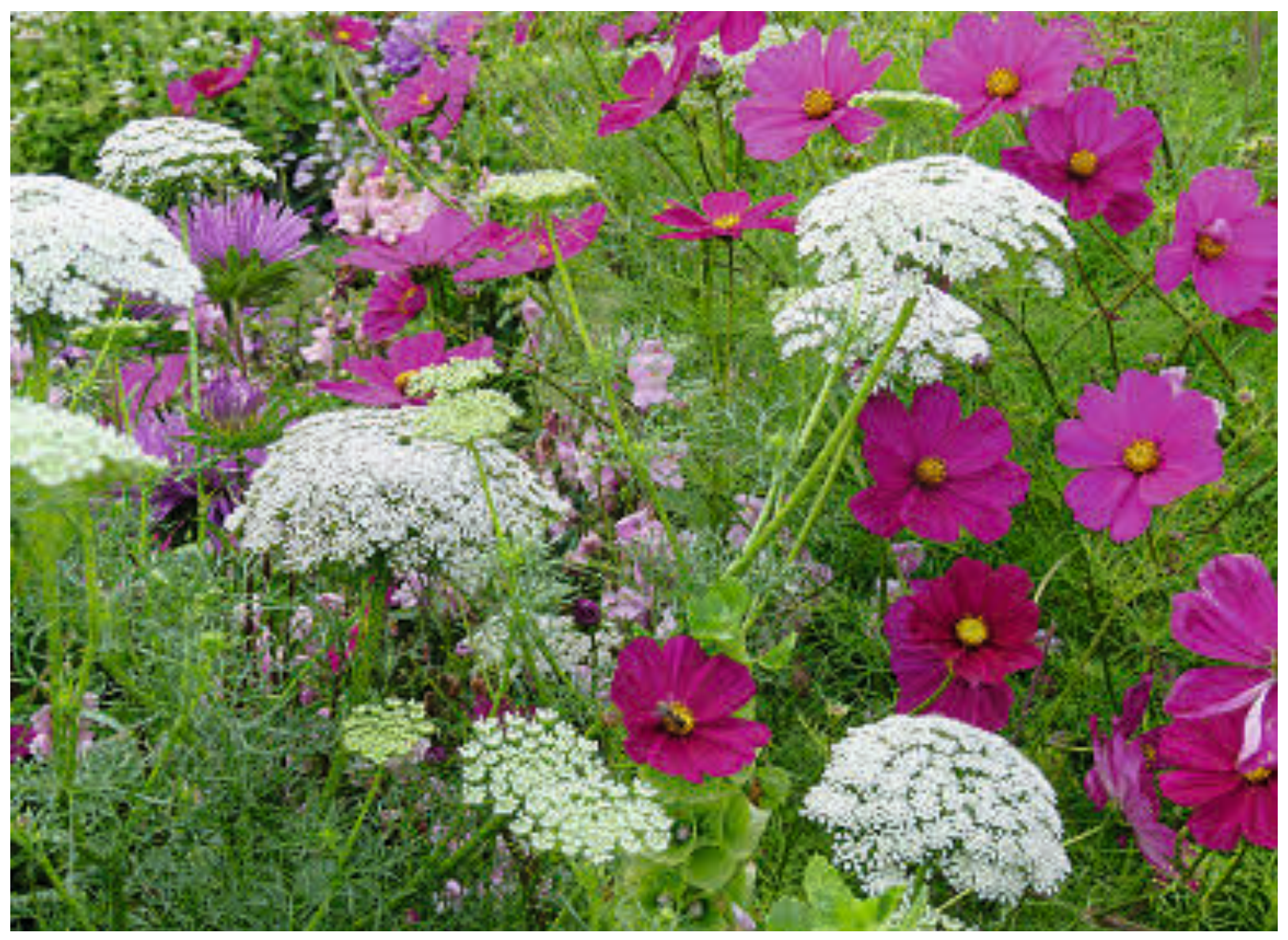

Wasser ist es ein ostpazifischer Delfin mit 252 Zähnen. Gar keine Zähne unter den Säugern haben Bartenwale, einige Schuppentiere und drei Arten von Ameisenbären. Unter den heute noch lebenden Säugern haben Elefanten die schwersten Zähne (Rekord: $117 \mathrm{~kg}$ pro Stoßzahn). Die härtesten Zähne haben Mäuse. Auf der bis 10 reichenden Mohs'schen Skala weisen sie einen Wert von 9,6 auf. Die Härte ihrer Zähne wird fast nur noch von Diamant (Härtegrad 10) übertroffen. Kein Wunder, dass Mäuse selbst die härtesten Fruchtschalen aufknabbern können. Zähne werden unterschiedlich oft getauscht. Der Mensch wechselt seine Zähne einmal im Leben. Der Elefant dagegen kann bis zu fünf Sätze an Mahlzähnen bilden, da diese durch das Zerkleinern von pflanzlicher Nahrung stark abgenutzt werden und nicht ein ganzes langes Elefantenleben funktionstüchtig bleiben würden. Haie ersetzen während ihres Lebens kontinuierlich ihre Zähne, die bei jedem Beutemachen dutzendweise verloren gehen.

\section{Pflanzen in der Zahnheilkunde}

Eine weitere Station war der Amberbaum ( $L i$ quidambar styraciflua). Dieser nordamerikanische Parkbaum wurde in seiner Heimat von den Indianern genutzt. Aus dem Stamm wurde traditionell ein flüssiges Balsamharz gewonnen, das die Indianer als natürliches Kaugummi zwischen die Zähne nahmen. Das Kauen auf dem aromatischen, antibakteriell wirkenden Holz diente der Zahnhygiene. Auch noch heute ist es Bestandteil industriell hergestellter Kaugummis. Zudem wird es zur Parfümierung von Seifen und Kosmetika verwendet. Weitere "Zahnbürstenbäume“ wurden vorgestellt, darunter der Niembaum (Azadirachta indica), auf dessen Zweigen in Indien als Ersatz für das Zähneputzen gekaut wird. Salvadora persica, ein in Afrika und Indien heimischer Baum aus der Familie der Salvadoraceae, wird ebenso Zahnbürstenbaum genannt. Der Name nimmt Bezug auf die Tradition, dass auf Wurzeln und Zweigen so lange gekaut wird, bis ein Ende bürstenartig ausgefranst ist und dann damit die 
Zähne geputzt werden können. Die Pflanze enthält u. a. Mineralstoffe, keimhemmend wirkende Substanzen und einen relativ hohen Fluorid-Anteil.

Weitere Pflanzen, die der Mundhygiene dienen oder gegen Zahnschmerzen verwendet werden, wurden vor allem in der Steppenwiese vorgestellt. Geeignet sind besonders Pflanzen mit ätherischen Ölen wie z. B. Salbei, Minze, Kamille, Estragon oder Majoran, ergänzend als tropische Art wurde die Gewürznelke genannt. Zerquetschte Blätter der Weinraute (Ruta graveolens) wurden in der Volksmedizin bei Zahnschmerzen in die hohlen Zähne gefüllt. Der leicht narkotisierende Milchsaft des häufig als Zierpflanze kultivierten Kalifornischen Goldmohns (Escholzia californica) wurde von den Ureinwohnern Nordamerikas zur Linderung von Zahnschmerzen eingesetzt. Blutwurz ist manchem vermutlich nur in Form eines flüssigen hochprozentigen Getränkes bekannt. Blutwurz (Potentilla erecta), eine Verwandte des Fingerkrauts, lässt sich gut an den nur vier statt sonst üblichen fünf Kronblättern erkennen und wächst bevorzugt auf Moor- und Heideböden. Aus den roten Wurzeln (daher der Name Blutwurz) lässt sich ein Tee aufbrühen, der z. B. für Mundspülungen bei Zahnschmerzen und Entzündungen im Rachenraum verwendet wurde.

Im Mittelalter war Vitamin-C-Mangel (Skorbut) eine gefürchtete Krankheit, die besonders im Winter oder bei Matrosen auf wochenlanger Schiffsfahrt auftrat. Die Krankheit äußert sich u.a. im Verlust von Zähnen. Erst im 18. Jh. wurde bekannt, dass der Konsum von Zitrusfrüchten gegen Skorbut hilft. Um dem Vitamin-

Abb. 5 (Seite 140): Die weißen Dolden des Zahnstocherkrautes (Ammi visnea) eignen sich gut für den Sommerflor.

Abb. 6 (oben): Aktiv ausgeschiedene Wassertropfen (Guttationstropfen) am gezähnten Blattrand des Frauenmantels (Alchemilla vulgaris).

Abb. 7 (Mitte): Gezähnter Blattrand einer Agave.

Abb. 8 (unten): Die Zähne an den wehrhaften Blattstielen der Doum-Palme (Hyphaene thebaica) erinnern an kleine Haifischzähne.
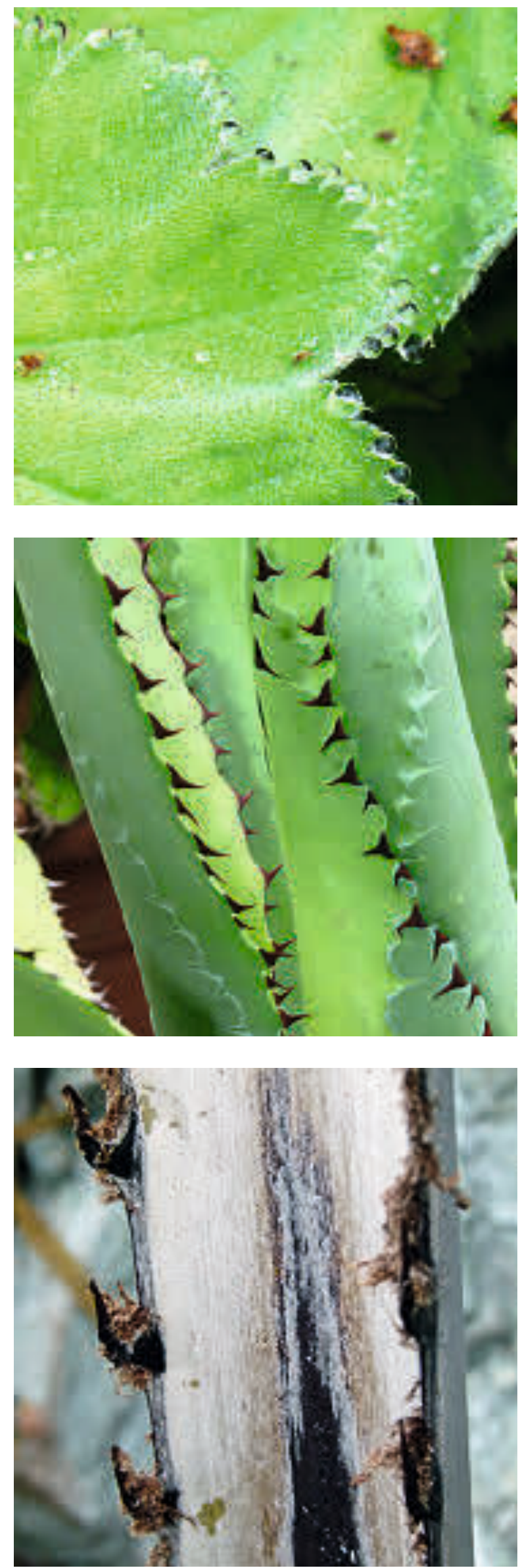

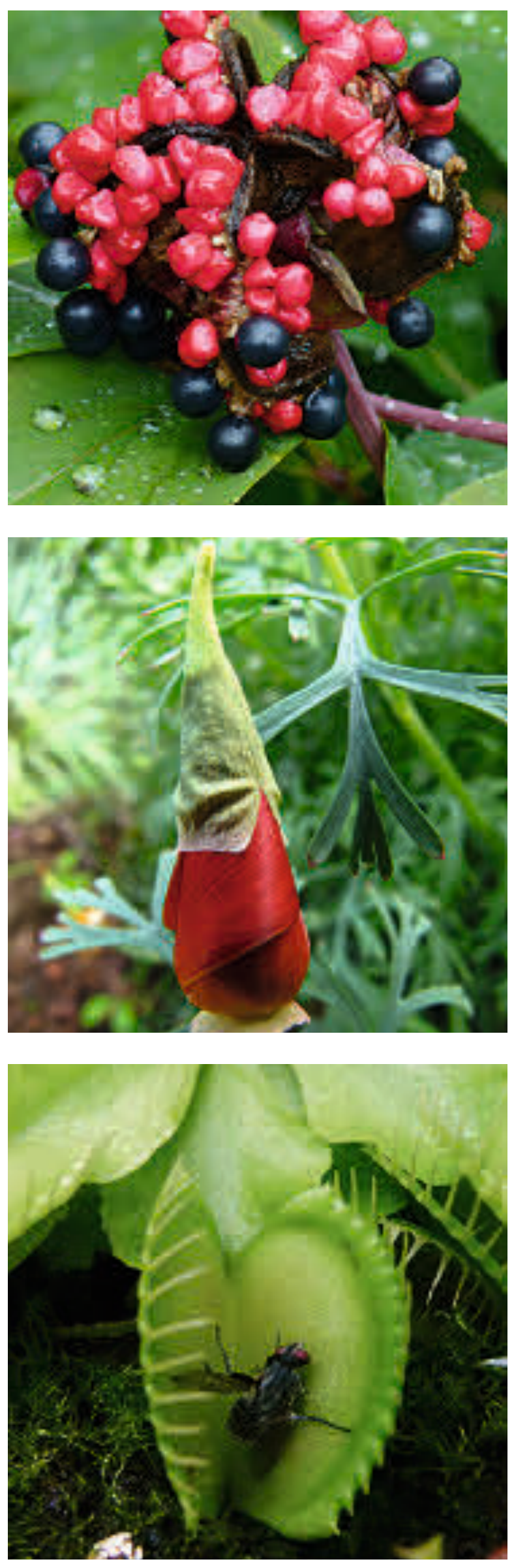

C-Mangel vorzubeugen, wurden deshalb auf längeren Schiffsreisen später neben Sauerkraut und Kartoffeln auch Zitronen bzw. Zitronensaft mit an Bord genommen. Von der täglichen Ration Limonensaft stammt der Spitzname Limey für die englischen Matrosen. Auf die Gepflogenheit, an Bord den Zitronensaft mit Rum gemischt zu trinken, gehen viele Cocktailrezepte zurück, die Rum oder Gin, gemischt mit Zitronensaft, enthalten.

Der Mythologie nach half auch Bilsenkraut (Hyoscyamus niger) bei diversen Zahnproblemen. Mittelalterliche Bader erhitzten die kleinen schwarzen Samen des Bilsenkrautes auf einer heißen Platte, so dass sie aufsprangen und ein kleiner heller, gekrümmter Embryo austrat. Dieser wurde den Patienten gezeigt. Es würde sich um den das Übel verursachenden Zahnwurm handeln, der nun entfernt sei. Der Glaube an den bösartigen Zahnwurm hat sich lange gehalten, es gibt viele Darstellungen dieses Plagegeistes.

\section{Tropische Zahnpflanzen}

Im Palmengarten sollten auch Palmen auf dem Führungsprogramm stehen. Die Samen verschiedener Palmen-Arten dienen als Ersatz für Elefanten-Elfenbein. Aus ihnen lassen sich kleine Figuren schnitzen oder beispielsweise Knöpfe anfertigen. Aus Ecuador stammt die Elfenbeinnusspalme (Phytelephas macrocarpa), in Afrika werden Samen verschiedener Hyphaene-

Abb. 9 (oben): Die schwarzen Samen von Pfingstrosen (hier Paeonia mlokosowitschii) galten früher als Mittel gegen Zahnschmerzen und wurden Zahnbohnen genannt.

Abb. 10 (Mitte): Mützenförmige Blütenknospe des Kalifornischen Goldmohnes (Escholzia californica), dessen leicht betäubender Milchsaft früher bei Zahnschmerzen verabreicht wurde.

Abb. 11 (unten): Fleischfressende Pflanzen wie die Venusfliegenfalle (Dionaea muscipula) benötigen keine Zähne.

Abb. 12 (Seite 143 links): Stoßzähne dienen Elefanten u.a. zum Graben.

Abb. 13 (Seite 143 rechts): Die spitzen Zähne des Hais sind typische Zähne eines Fleischfressers. 


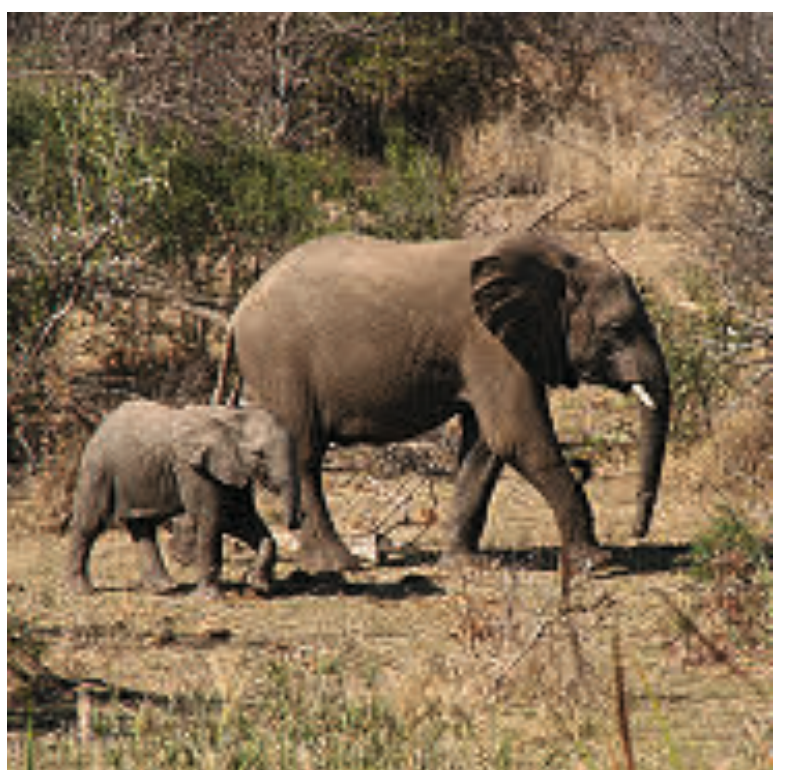

Arten verarbeitet. Auch Raphia- oder Betelpalmen-Samen werden im Drechslerbedarf angeboten.

Meist schon im Dämmerlicht führte der zweite Abschnitt der Veranstaltung durch das Tropicarium. Dort gab es den Kautschukbaum (Hevea brasiliensis) zu sehen. Naturkautschuk ist vielseitig verwendbar, u.a. auch für medizinische Einmalhandschuhe, die auch aus Zahnarztpraxen nicht wegzudenken sind. Der Guttaperchabaum (Pallaquium gutta) liefert ebenfalls kautschukartiges Material für die Zahnmedizin. Es kommt hauptsächlich bei Wurzelkanalbehandlungen zum Einsatz. Die zum Füllen der Kanäle verwendeten so genannten Guttaperchaspitzen enthalten neben einer Reihe anderer Bestandteile einen hohen Prozentsatz an Guttapercha. Auch für provisorische Füllungen ist Guttapercha geeignet, da es sich in einem Stück wieder entfernen lässt. Es gibt verschiedene Pflanzen, die aufgrund ihres zähen Milchsaftes Guttaperchabaum genannt werden, so auch Eucommia ulmoides, ein Laubbaum aus China, der bei uns im Freien kultiviert werden kann. Werden die Blätter quer in viele Segmente auseinander gerissen, fallen, wie von Zauberhand gehal-

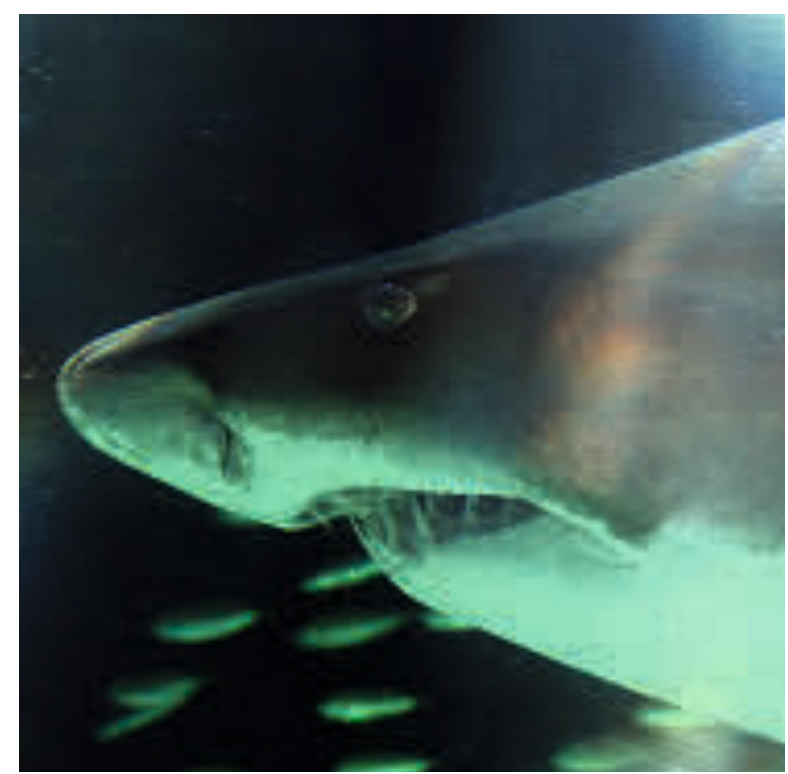

ten, die einzelnen Blattabschnitte nicht zu Boden. Die dünnen, durchsichtigen und klebrigen Latexfäden, die die Stücke zusammenhalten, sind mit bloßem Auge so gut wie nicht zu erkennen. Jeder Teilnehmer bekam ein Blatt, um die "Zerreißprobe“ auszutesten.

Süßen ohne Karies zu verursachen oder dick zu machen, ist mit den Blättern der SüßblattPflanze (Stevia rebaudiana) möglich. Die Teilnehmer konnten sich von der enormen Süßkraft frischer Blattstückchen dieses tropischen Korbblütlers überzeugen, die um ein Vielfaches höher als bei normalem Haushaltszucker ist.

Häufig wird die Frage gestellt, ob Fleischfressende Pflanzen auch Zähne hätten, weshalb Karnivoren mit in das Programm aufgenommen wurden. Die Fallentypen von Venusfliegenfalle (Dionaea muscipula) und Kannenpflanze (Nepenthes spec.) wurden erläutert. Zum Abschluss ging es in die Halbwüste zu Tigerrachen (Faucaria tuberculosa). Zudem wurde ein Text über einen schmerzenden Löwen-Zahn vorgetragen. Nach gut 1,5 Stunden schließlich war die Führung beendet und manch einer hatte einen „Affenzahn drauf“, um die nächste UBahn nicht zu verpassen. 